\title{
Analytical Computation of End-Coil Leakage Inductance of Round- Rotor Synchronous Machines Field Winding
}

\author{
Mauro Bortolozzi*, Alberto Tessarolo*, IEEE, Senior Member, and Claudio Bruzzese ${ }^{\circ}$, IEEE, Member \\ * Engineering and Architecture Department, University of Trieste, 34127 Trieste, Italy \\ ○Astronautical, Electrical and Energy Engineering Department, University of Rome "Sapienza", Rome, Italy
}

\begin{abstract}
The computation of end coil leakage inductances of electric machines is a challenging task due to the complicated leakage flux 3D distribution in the winding overhang region. In this paper the problem is addressed of computing the field circuit leakage inductance of round-rotor synchronous machines. The proposed method is fully analytical and descends from the symbolical solution of Neumann integrals applied to the computation of self and mutual inductances combined with the method of mirror images to account for core effects. With respect to existing analytical approaches, the methodology requires neither numerical integral solutions nor discretizing the end-coil geometry into small straight elements. The accuracy of the proposed technique for computing the mutual inductance between two single end turns is assessed against measurements on a dedicated experimental set-up. The extension of the method to the computation of the entire field-circuit end-coil leakage inductance is assessed by comparison with 3D finite element analysis (FEA).
\end{abstract}

Index Terms-Analytical methods, field windings, leakage inductance computation, method of mirror images, Neumann integrals, round-rotor synchronous machines.

\section{INTRODUCTION}

$\mathrm{T}$ HE computation of end-coil leakage inductance is a challenge in the analysis and simulation of electric machines due to the complicated 3D distribution of leakage fluxes in the winding overhang region. Such flux distribution can be studied using 3D Finite Element Analysis (FEA) which, however, is a very resource-consuming method [1][2]. Some authors have tried to work out a parametric calculation approach for concentrated-coil end-turn leakage inductance computation based on a large amount of 3D and 2D FEA simulations [3]. Purely analytical-numerical algorithms, not requiring any FEA, have been proposed in the literature to estimate end coil leakage inductances of distributed windings based on solving Neumann integrals for mutual inductance computation [4] combined with the method of mirror images [5] to account for magnetic core effects [6][10]. When applied to the coil geometry of usual stator distributed windings, Neumann integrals do not allow for a symbolical solution [6]-[8]; their computation requires approximating each end coil as a set of elementary straight filaments [7]-[9], for which a closed-form solution of Neumann integrals is known [11]. This constitutes a significant complication because the mutual inductance between all pairs of coils results in the sum of a large number of terms [7]-[8]. Conversely, as theoretically investigated in [10], a fully-analytical solution to Neumann integrals can be derived when computing the end-coil leakage inductances of distributed concentric windings. In this paper, the analytical formulations proposed in [10] are firstly verified by measurements on a small-scale laboratory setup; secondly, the validated equations are applied to the end-coil leakage inductance computation of the field windings in cylindricalrotor synchronous machines [7], [12]. The method is, in particular, applied to a 4-pole 16-MVA medium-voltage wound-field round-rotor synchronous machine and analytical results are assessed by comparison with 3D FEA showing a satisfactory accordance. With respect to other computation techniques for end-coil leakage inductance, the proposed approach is very fast and flexible as it does not require any detailed geometry modeling, end-coil shape discretization and numerical integral solutions.

The paper is organized as follows. In Section II the analytical formula for mutual inductance computation between two generic end-coils in a round-rotor synchronous machine field winding are reported. In Section III, these formulas are assessed both by measurements on a dedicated experimental equipment and by 3D FEA. In Section IV, the validated formulas are applied to leakage inductance computation of the entire field winding. In Section V, an application case study is reported referring to a 16-MVA synchronous machine for which analytical results are compared to 3D FEA computations. Finally, Section VI discusses the effect of a ferromagnetic shaft on the field winding end coil inductance.

\section{ANALYTICAL FORMULA FOR COMPUTING THE MUTUAL INDUCTANCE BETWEEN TWO END COILS}

The typical shape of the field circuit end coils for a roundrotor synchronous machine is illustrated in Fig. 1.

\section{A. End coil geometric modeling}

As a first step for determining the leakage inductance of this kind of end windings is the analytical calculation of the mutual inductance for two generic end turns, modeled as ideal filaments with infinitesimal cross section as shown in Fig. 2. The geometric model of the two end turns is represented in Fig. 2 with solid lines (curves $\mathrm{ABCD}, \mathrm{A}^{\prime} \mathrm{B}^{\prime} \mathrm{C}^{\prime} \mathrm{D}^{\prime}$, respectively), while dashed lines indicate fictitious conductors that need to be introduced for the mutual inductance computation through the method of mirror images. The approach followed to
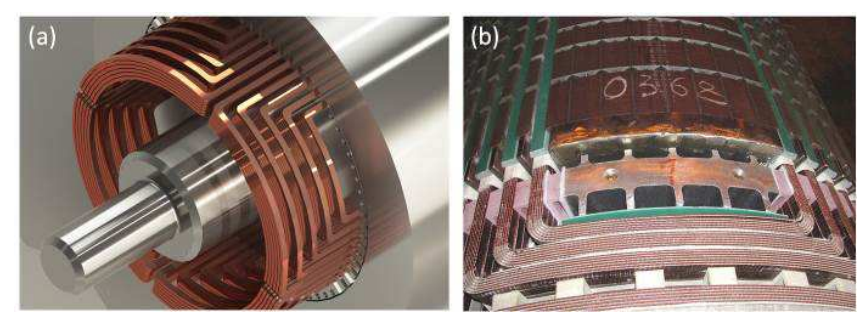

Fig. 1. Field winding details of round-rotor synchronous machines (a) 3D model; (b) picture taken on a real machine during manufacturing. 


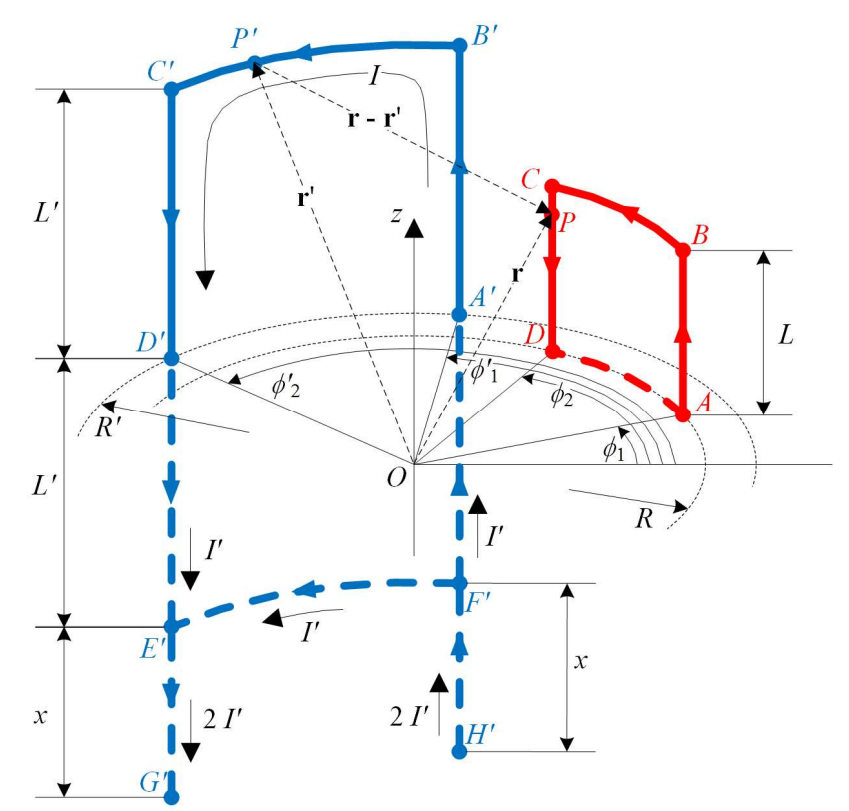

Fig. 2. Geometric modeling of two end coils (solid lines). Dashed lines represent fictitious conductors used for mutual inductance computation.

compute the mutual inductance is to impress a current $I$ in one end turn $\left(\mathrm{A}^{\prime} \mathrm{B}^{\prime} \mathrm{C}^{\prime} \mathrm{D}^{\prime}\right)$ and to compute the flux $\lambda$ linked by the other end turn, i.e. the flux linked by the closed loop ABCD. The mutual inductance is hence derived as $\lambda / I$. In order to account for stator and rotor magnetic core effects through the method of mirror images, the finite fictitious conductors $\mathrm{D}^{\prime} \mathrm{E}^{\prime}$, $\mathrm{E}^{\prime} \mathrm{F}^{\prime}$ and $\mathrm{A}^{\prime} \mathrm{F}^{\prime}$, carrying the current $I^{\prime}$, are introduced [5], [7]. Moreover, the further fictitious conductors $\mathrm{E}^{\prime} \mathrm{G}^{\prime}$ and $\mathrm{F}^{\prime} \mathrm{H}^{\prime}$, carrying the current $2 I^{\prime}$, are used such that their length $x$ tends to infinity. The value of the ideal current $I^{\prime}$ depends on the relative magnetic permeability $\mu_{r}$ of the stator and rotor cores [5]. In the following of the paper the assumption will be made that the iron cores have infinite magnetic permeability with respect to the air and this hypothesis leads to set $I^{\prime}=I$ [5].

The reason for adding the fictitious conductors shown in Fig. 2 and the justification for the currents flowing through them are extensively explained in [5], where the method of mirror images is applied to the computation of electric machine end winding leakage inductance.

The infinitely permeable stator and rotor cores are approximated as the semi-space $z<0$ in the cylindrical coordinate system with origin $O$ as shown in Fig. 2 In such coordinate system, the proposed geometric modeling makes it possibly to univocally identify the shape of the two generic end coils through the set of parameters $\phi_{1}, \phi_{2}, R, L$ and $\phi_{1}^{\prime}, \phi_{2}^{\prime}$, $R^{\prime}, L^{\prime}$; respectively hence the mutual inductance between these end coils will be indicated as $M_{\left(\phi_{1}, \phi_{2}, L, R\right)-\left(\phi_{1}^{\prime}, \phi_{2}^{\prime}, L^{\prime}, R^{\prime}\right)}$.

\section{B. Analytical expression through Neumann integrals}

Making use of Neumann integrals applied to the geometric model shown in Fig. 2 leads to the following expression of the mutual inductance between the two end coils:

$$
M_{\left(\phi_{1}, \phi_{2}, L, R\right)-\left(\phi_{1}^{\prime}, \phi_{2}^{\prime}, L^{\prime}, R^{\prime}\right)}=
$$

$$
\begin{aligned}
& \frac{\mu_{0}}{4 \pi}\left\{\oint_{A B C D} \int_{A^{\prime} B^{\prime} C^{\prime} D^{\prime}} \frac{d \mathbf{r} \times d \mathbf{r}^{\prime}}{\left|\mathbf{r}-\mathbf{r}^{\prime}\right|}+\oint_{A B C D} \int_{D^{\prime} E^{\prime}} \frac{d \mathbf{r} \times d \mathbf{r}^{\prime}}{\left|\mathbf{r}-\mathbf{r}^{\prime}\right|}\right. \\
& +\oint_{A B C D} \int_{F^{\prime} A^{\prime}} \frac{d \mathbf{r} \times d \mathbf{r}^{\prime}}{\left|\mathbf{r}-\mathbf{r}^{\prime}\right|}+\oint_{A B C D} \int_{F^{\prime} E^{\prime}} \frac{d \mathbf{r} \times d \mathbf{r}^{\prime}}{\left|\mathbf{r}-\mathbf{r}^{\prime}\right|}+ \\
& \left.+2 \oint_{A B C D} \int_{E^{\prime} G^{\prime}} \frac{d \mathbf{r} \times d \mathbf{r}^{\prime}}{\left|\mathbf{r}-\mathbf{r}^{\prime}\right|}+2 \oint_{A B C D} \int_{H^{\prime} F^{\prime}} \frac{d \mathbf{r} \times d \mathbf{r}^{\prime}}{\left|\mathbf{r}-\mathbf{r}^{\prime}\right|}\right\} .
\end{aligned}
$$

In (1), $\mathbf{r}$ and $\mathbf{r}^{\prime}$ are the radius vectors that identify two generic points lying on the two end turn models as shown in Fig. 2 and integrals are performed over oriented paths (e.g. $\mathrm{D}^{\prime} \mathrm{E}^{\prime}$ indicates the oriented segment which begins at $\mathrm{D}^{\prime}$ and end at $\left.\mathrm{E}^{\prime}\right)$.

Equation (1) can be rewritten as follows:

$$
m_{i j}=\frac{\mu_{0}}{4 \pi}\left(N_{s t r}+N_{\text {arc }}+N_{i n f}\right)
$$

where: $N_{s t r}$ is the sum of Neumann integrals including straight segments of finite length; $N_{\text {arc }}$ is the sum of Neumann integrals including circular arcs; and $N_{i n f}$ is the sum of Neumann integrals including straight segments whose length tends to infinity; in symbols:

$$
\begin{gathered}
N_{s t r}=\int_{A B \cup C D} \int_{F^{\prime} B^{\prime} \cup C^{\prime} E^{\prime}} \frac{d \mathbf{r} \times d \mathbf{r}^{\prime}}{\left|\mathbf{r}-\mathbf{r}^{\prime}\right|}, \\
N_{a r c}=\int_{B C \cup D A} \int_{B^{\prime} C^{\prime} \cup F^{\prime} E^{\prime}} \frac{d \mathbf{r} \times d \mathbf{r}^{\prime}}{\left|\mathbf{r}-\mathbf{r}^{\prime}\right|}, \\
N_{\text {inf }}=2 \int_{A B \cup C D} \int_{E^{\prime} G^{\prime} \cup H^{\prime} F^{\prime}} \frac{d \mathbf{r} \times d \mathbf{r}^{\prime}}{\left|\mathbf{r}-\mathbf{r}^{\prime}\right|} .
\end{gathered}
$$

In the following, a closed-form analytical expression for the terms (3)-(5) is provided based on the mathematical derivations detailed in [10].

\section{1) Term accounting for finite straight elements}

The term $N_{\text {str }}$ can be written as [10]:

$$
N_{s t r}=n_{s t r}\left(\phi_{1}, \phi_{1}^{\prime}\right)+n_{s t r}\left(\phi_{2}, \phi_{2}^{\prime}\right)-n_{s t r}\left(\phi_{1}, \phi_{2}^{\prime}\right)-n_{s t r}\left(\phi_{2}, \phi_{1}^{\prime}\right) \text {, }
$$

where the $n_{s t r}(x, y)$ is defined as per (7) with the function $d(x, y)$ given by (8):

$$
\begin{aligned}
n_{s t r}(x, y) & =\sum_{k=-1,1}\left\{k\left(L^{\prime}+k L\right) \operatorname{asinh}\left(\frac{L^{\prime}+k L}{d(x, y)}\right)-\right. \\
& \left.-k \sqrt{\left(L^{\prime}+k L\right)^{2}+d(x, y)^{2}}\right\} \\
d(x, y) & =\sqrt{R^{2}+R^{\prime 2}-2 R R^{\prime} \cos (x-y)} .
\end{aligned}
$$

2) Term accounting for circular arcs

The term $N_{\text {arc }}$ can be expressed as [10]:

$$
\begin{gathered}
N_{\text {arc }}=n_{\text {arc }}\left(L, \theta, \theta^{\prime}\right)+n_{\text {arc }}\left(L, \theta, \pi-\theta^{\prime}\right)- \\
-n_{\text {arc }}\left(0, \frac{\pi}{2}, \theta^{\prime}\right)-n_{\text {arc }}\left(0, \frac{\pi}{2}, \pi-\theta^{\prime}\right),
\end{gathered}
$$

where the function $n_{\text {arc }}(\ell, x, y)$ is defined by (10) and the angles $\theta, \theta^{\prime}$ are given by (11).

$$
n_{\text {arc }}(\ell, x, y)=
$$




$$
\begin{gathered}
=\frac{R R^{\prime}}{r_{>}(\ell)}\left\{U+\sum_{n=1}^{+\infty}\left[\frac{r_{<}(\ell)}{r_{>}(\ell)}\right]^{n} \sum_{m=0}^{n} K_{n, m} P_{n}^{m}(\cos (x)) P_{n}^{m}(\cos (y)) V_{m}\right\} \\
\theta=\operatorname{atan} 2(L, R), \theta^{\prime}=\operatorname{atan} 2\left(L^{\prime}, R^{\prime}\right)
\end{gathered}
$$

In the definition (10), $P_{n}^{m}(x)$ are the associated Legendre polynomials [13]:

$$
\begin{gathered}
P_{n}^{m}(x)= \\
=\frac{\left(1-x^{2}\right)^{m / 2}}{2^{n}} \sum_{k=0}^{\text {floor }(n / 2)}(-1)^{k+m} m !\left(\begin{array}{l}
n \\
k
\end{array}\right)\left(\begin{array}{c}
2 n-2 k \\
n
\end{array}\right)\left(\begin{array}{c}
n-2 k \\
m
\end{array}\right) x^{n-2 k-m},
\end{gathered}
$$

the functions $r_{<}(\ell), r_{>}(\ell)$ are given by:

$$
\begin{aligned}
& r_{>}(\ell)=\max \left(\sqrt{R^{2}+\ell^{2}}, \sqrt{R^{\prime 2}+L^{\prime 2}}\right) \\
& r_{<}(\ell)=\min \left(\sqrt{R^{2}+\ell^{2}}, \sqrt{R^{\prime 2}+L^{\prime 2}}\right)
\end{aligned}
$$

and the constants $U, V_{m}, K_{m, n}$ are defined as follows:

$$
\begin{gathered}
K_{n, m}= \begin{cases}1 / 2 & \text { if } m=0 \\
(n-m) ! /(n+m) ! & \text { if } m \neq 0\end{cases} \\
U=\sum_{j=0,1} \sum_{k=0,1}(-1)^{j+k} \cos \left(c_{j, k}\right) \\
V_{m}= \begin{cases}\sum_{j=0,1} \sum_{k=0,1} \sum_{q=-1,1}(-1)^{j+k} \frac{\cos \left[c_{j, k}(m+q)\right]}{(m+q)^{2}} & \text { if } m \neq 1 \\
\sum_{j=0,1} \sum_{k=0,1}(-1)^{j+k}\left[\frac{\cos ^{2}\left(c_{j, k}\right)}{2}+b_{j, k}\right] & \text { if } m=1\end{cases}
\end{gathered}
$$

with

$$
\begin{aligned}
c_{j, k} & =\phi_{1}+j\left(\phi_{2}-\phi_{1}\right)-\left[\phi_{1}^{\prime}+k\left(\phi_{2}^{\prime}-\phi_{1}^{\prime}\right)\right], \\
b_{j, k} & =\left[\phi_{1}+j\left(\phi_{2}-\phi_{1}\right)\right]\left[\phi_{1}^{\prime}+k\left(\phi_{2}^{\prime}-\phi_{1}^{\prime}\right)\right] .
\end{aligned}
$$

3) Term accounting for infinite straight segments The term $N_{\text {inf }}$ can be written as [10]:

$$
N_{\text {inf }}=2\left[n_{\text {inf }}\left(\phi_{1}, \phi_{1}^{\prime}, \phi_{2}^{\prime}\right)-n_{\text {inf }}\left(\phi_{2}, \phi_{2}^{\prime}, \phi_{1}^{\prime}\right)\right],
$$

where the function $n_{\text {inf }}(x, y, z)$ is defined as follows:

$$
\begin{gathered}
n_{\text {inf }}(x, y, z)=L \ln \left[\frac{d(x, z)}{d(x, y)}\right]+ \\
\sum_{k=0,1}\left\{(-1)^{k}\left(k L+L^{\prime}\right)\left[\operatorname{asinh}\left(\frac{L^{\prime}+k L}{d(x, y)}\right)-\operatorname{asinh}\left(\frac{L^{\prime}+k L}{d(x, z)}\right)\right]+\right. \\
\left.-(-1)^{k}\left[\sqrt{\left(k L+L^{\prime}\right)^{2}+d(x, y)^{2}}-\sqrt{\left(k L+L^{\prime}\right)^{2}+d(x, z)^{2}}\right]\right\} .
\end{gathered}
$$

\section{VALIDATION THROUGH 3D FEA AND BY EXPERIMENTAL MEASUREMENTS}

In the previous Section, the fully analytical formulas have been provided to compute the mutual inductance $M_{\left(\phi_{1}, \phi_{2}, L, R\right)-\left(\phi_{1}^{\prime}, \phi^{\prime}, L^{\prime}, R^{\prime}\right)}$ between two infinitely-thin end turns characterized by parameters $\phi_{1}, \phi_{2}, L, R$ and $\phi_{1}^{\prime}, \phi_{2}^{\prime}, L^{\prime}, R^{\prime}$,

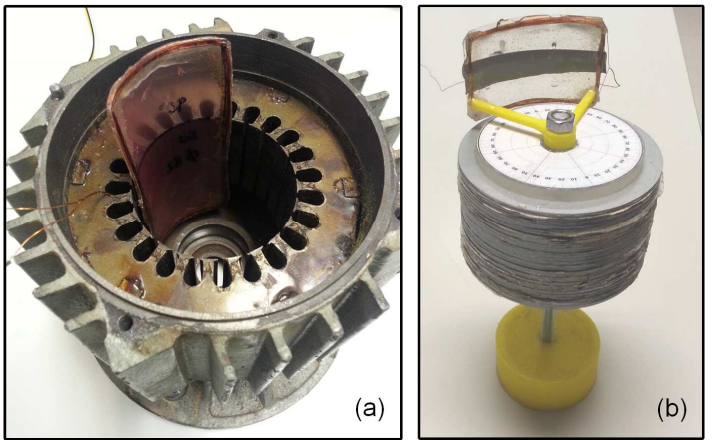

Fig. 3. (a) Stator core and frame with the search coil fixed to the stator bore; (b) Dummy rotor core with the search coil fixed on its axis.
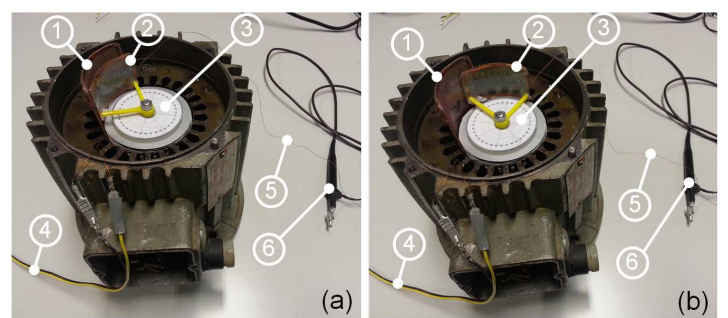

Fig. 4. Experimental setup for the verification of mutual inductance calculation between end coils: 1-source coil; 2-search coil; 3-graduated angular scale; 4-supply terminals of the source coil; 5 - twisted terminals of the search coil; 5-voltage probe. The figure shows the equipment for two different positions (a) and (b) of the search coil.

respectively, as illustrated in Fig. 2. In this Section, the mentioned formulas are assessed with two independent approaches, namely: by experiments on a dedicated laboratory set-up and by 3D FEA.

\section{A. Experimental validation}

The experimental setup is built using the stator of an induction motor with a source coil (including $N^{\prime}$ turns) fixed to the stator bore as shown in Fig. 3a (the characteristic data $N^{\prime}$, $\phi_{1}^{\prime}, \phi_{2}^{\prime}, L^{\prime}, R^{\prime}$ of the source coil are given in Table I). A dummy rotor, made of a laminated silicon steel stack, is also built and a search coil (including $N$ turns) is connected to its axis as shown in Fig. 3b. The air gap width is $2 \mathrm{~mm}$.

TABLE I. CHARACTERISTIC DIMENSIONS OF THE END COILS USED FOR
EXPERIMENTAL VALIDATIONS
\begin{tabular}{cc|cc}
\multicolumn{2}{c}{ Source coil } & \multicolumn{2}{c}{ Search coil } \\
\hline \hline$L^{\prime}$ & $48 \mathrm{~mm}$ & $L$ & $35 \mathrm{~mm}$ \\
\hline$R^{\prime}$ & $41 \mathrm{~mm}$ & $R$ & $40 \mathrm{~mm}$ \\
\hline$\phi_{2}^{\prime}-\phi_{1}^{\prime}$ & $90^{\circ}$ & $\phi_{2}-\phi_{1}$ & $80^{\circ}$ \\
\hline$N^{\prime}$ & 5 & $N$ & 20
\end{tabular}

The search coil (whose characteristic data $N, \phi_{1}, \phi_{2}, L, R$ are given in Table I), can freely rotate around the rotor axis.

The entire assembly of the experimental setup is shown in Fig. 4 for two possible angular positions of the search coil. From Fig. 4 it can be also seen how the angular position of the search coil can be precisely identified by means of an angular graduated scale fixed to the dummy rotor stack. 


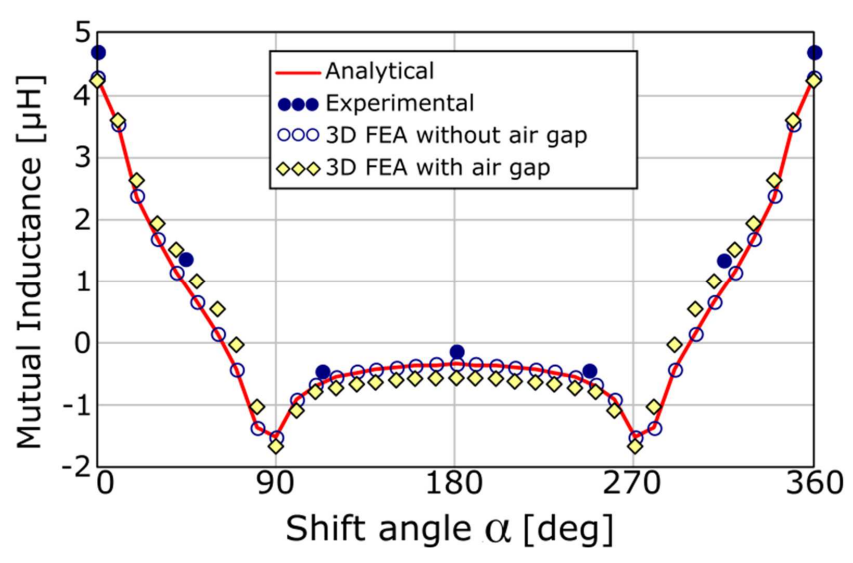

Fig. 5. Comparison between mutual inductance values obtained analytically, experimentally and by 3D FEA.

For the experimental validation, the source coil is energized with a sinusoidal current $I$ at a frequency $f=50 \mathrm{~Hz}$ and the electromotive force (EMF) $E$ induced between the search coil terminals is measured. The measurement is repeated for various displacement angles $\alpha$ between the source and search coil axes and, for each measurement, the mutual inductance between the two end coils is computed as follows:

$$
M_{\text {test }}(\alpha)=\frac{E}{2 \pi f I} .
$$

The measurement is compared to the analytical calculation, that is:

$$
M_{\text {analyt }}(\alpha)=M_{\left(\phi_{1}+\alpha, \phi_{2}+\alpha, L, R\right)-\left(\phi_{1}^{\prime}, \phi_{2}^{\prime}, L^{\prime}, R^{\prime}\right)} \times N \times N^{\prime}
$$

where $N$ and $N^{\prime}$ are the number of turns for the search and source coils respectively.

The result of the comparison between the analytical and measurement results is provided in Fig. 5 showing a satisfactory accordance.

\section{B. 3D FEA validation}

A further validation is performed using 3D FEA based on the meshed model shown in Fig. 6. The two end coils used in the experiment (Fig. 3 and Fig. 4) emerge from a circular ferromagnetic slab of ideally infinite relative magnetic permeability $\left(\mu_{r}=2 \times 10^{5}\right)$ which represents the stator and rotor cores. While the source coil passes through the ferromagnetic slab, the search coil is modeled as a closed loop by adding the fictitious arc identified by "3" in Fig. 6. The source coil (composed of $N^{\prime}$ turns) is then energized with a current $I$, while the flux $\lambda$ linked by the search coil (composed of $N$ turns) is computed by $3 \mathrm{D}$ FEA. The computation is repeated for different shift angles $\alpha$ between the two end coil axes and, in each case, the mutual inductance is derived as:

$$
M_{F E A}(\alpha)=\frac{\lambda}{I} \text {. }
$$

Furthermore, the simulation is performed both on a model without air gap (Fig. 6a-b) and on a model including an air gap with different possible widths (Fig. 6c), in order to investigate the air gap influence on inductance calculation.

The mutual inductance as a function of the shift angle $\alpha$ computed by 3D FEA on the model with no air gap and on a model with $3 \mathrm{~mm}$ air-gap width is plotted in Fig. 5 and can be
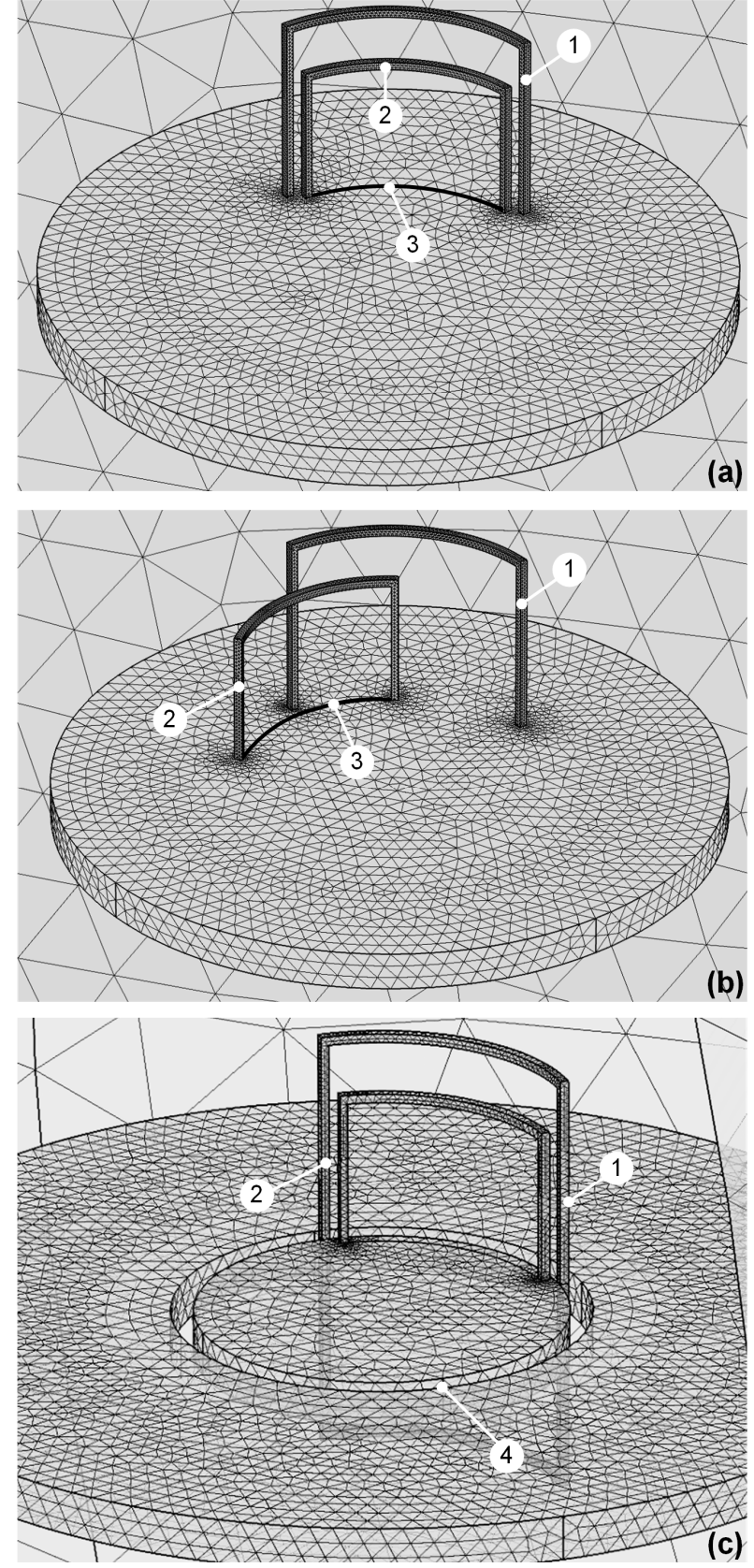

Fig. 6. 3D model for FEA validation. 1-source coil; 2-search coil; 2-fictitious arc needed to make the search end coil a closed loop. 4-Air gap. (a) and (b) show the model with no air gap for two different shift angles $\alpha$ between the end coils; (c) shows the model with a 3-mm air gap.

seen to be in good agreement with the analytical prediction. The air gap actually affects mutual inductance values as it alters the spatial end-coil leakage field distribution, but the resulting errors are such that they do not invalidate the accuracy of the computation. By reducing the air gap width $g$ in the 3D FEA model (in the experimental set-up $g=2 \mathrm{~mm}$ ), the results obtained by 3D FEA tend to converge to the analytically computed values, up to practically coinciding with them when the air gap vanishes (Fig. 5).

The possibility to disregard air-gap effects while preserving an acceptable accuracy in end coil leakage inductance prediction is confirmed by various works [5], [7], 
[8] where, in spite of this approximation, a good accordance is achieved between measurements and calculations. Interesting attempts to include air-gap effects by means on suitable "virtual air-gap conductors" appear in some recent works [9], although lacking assessment by either experiments or 3D FEA simulations. The introduction of virtual air-gap conductors could be a possible way to further increase the accuracy of the method in future researches, but currently stands outside the scope of this paper.

\section{INDUCTANCE COMPUTATION FOR THE ENTIRE FIELD WINDING}

In this Section the problem is addressed to determine the end coil inductance of the entire field winding for a roundrotor synchronous machine with $P$ poles. The structure of the field winding is illustrated in Fig. 7 where it can be seen that each of the $P$ poles includes $K$ coils of different size. Each end coil is denoted as $\mathrm{C}_{p, k}$ (with $p=1 . . P$ and $k=1 . . K$ ) and is composed of $N_{k}$ series-connected turns, each of length $l_{k}$.

The total end coil inductance $L_{t o t}$ of the winding can be computed as follows [4]:

$$
L_{t o t}=2\left[P \sum_{k=1 . . K} L_{k}+\sum_{\begin{array}{r}
p, p^{\prime}=1 . . P \\
p \neq p^{\prime}
\end{array}} \sum_{\substack{k, k^{\prime}=1 . . K \\
k \neq k^{\prime}}}(-1)^{p+p^{\prime}} M_{p, k, p^{\prime}, k^{\prime}}\right],
$$

where: the coefficient 2 is used to account for end coils at both rotor ends, $L_{k}$ is the self-inductance of coil $\mathrm{C}_{p, k}$ (which is independent of $p$ ) and $M_{p, k, p^{\prime}, k^{\prime}}$ is the mutual inductance between two generic distinct coils $\mathrm{C}_{p, k}$ and $\mathrm{C}_{p^{\prime}, k^{\prime}}$. The coefficient $(-1)^{p+p^{\prime}}$ accounts for the fact that the field current reverses when we move from one field pole to the adjacent one.

\section{A. Self-inductance of an end coil}

The self-inductance consists of two terms:

$$
L_{k}=L_{k}^{(i n t)}+L_{k}^{(e x t)},
$$

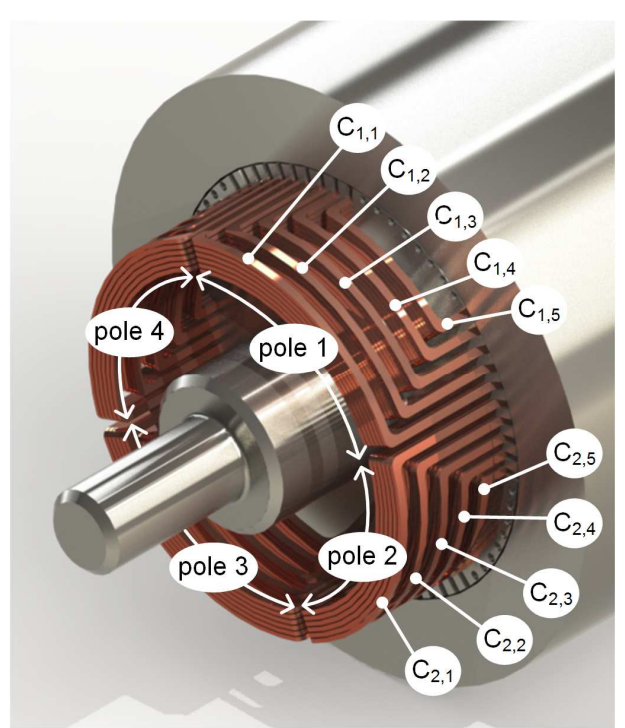

Fig. 7. Illustration of a 4-pole field winding with five coils per pole. $C_{p, k}$ indicates the $k$-th coil of the $p$-th pole. where $L_{k}^{(i n t)}$ is the "internal inductance" [7] due to the flux crossing the conductors, and $L_{k}^{(e x t)}$ is the "external inductance" due to the flux linked by the coil but not crossing the conductors. Following [7], the internal inductance is computed approximating the coil as a circular conductor, so that $L_{k}^{(i n t)}$ is independent of the coil cross section and only depends on the end coil length $l_{k}$ as follows:

$$
L_{k}^{(i n t)}=N_{k}^{2} \frac{\mu_{0}}{8 \pi} \cdot l_{k} .
$$

where the end coil length is easily computed based on its dimensions $L, R$ and $\phi_{1}-\phi_{2}$.

As regards the external self-inductance of the generic end coil $C_{p, k}$, it is computed following the procedure described in [8]. For this purpose, in each end coil $C_{p, k}$ two curves $\Gamma_{p, k}$ and $\Lambda_{p, k}$ are defined as illustrated in Fig. 8 and Fig. $9 . \Gamma_{p, k}$ is an open curve developing along the mean path of the end coil (Fig. 8b), while $\Lambda_{p, k}$ is a closed loop that unfolds along the internal periphery of the end coil (Fig. 8b, Fig. 9). The internal inductance is then computed using the algorithms explained in

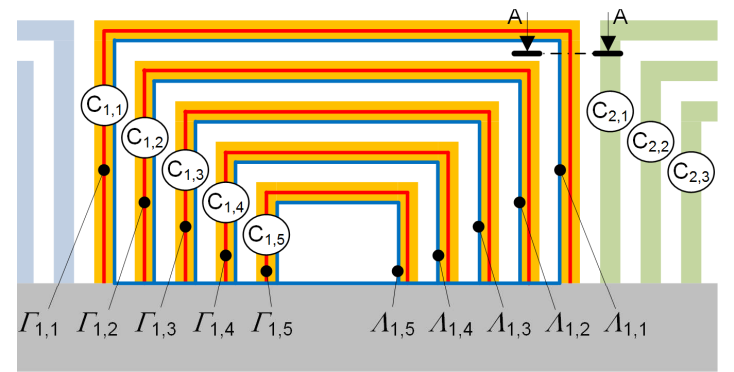

(a)

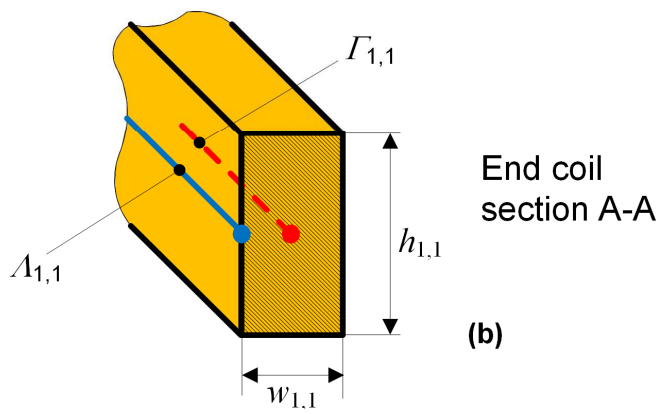

Fig. 8. (a) End coils $\mathrm{C}_{11}, . ., \mathrm{C}_{1,5}$ of pole 1 and relevant curves $\Gamma_{11}, . ., \Gamma_{1,5}$ and $\Lambda_{11}, \ldots, \Lambda_{1,5}$ (b) Section A-A of end-coil $\mathrm{C}_{1,1}$ illustrating the position of the auxiliary curves $\Gamma_{1,1}$ and $\Lambda_{1,1}$.

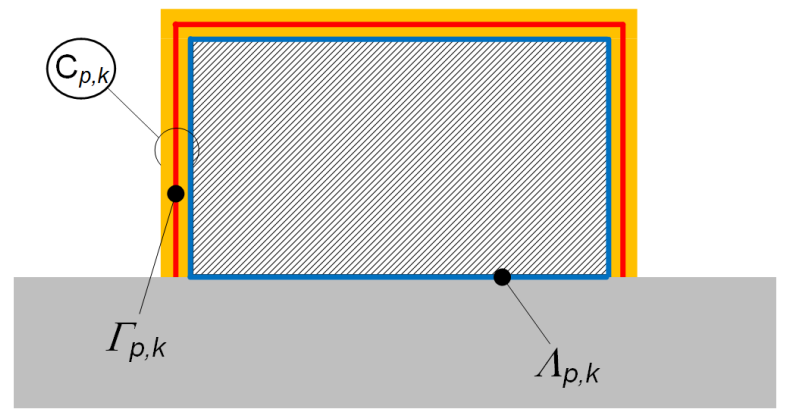

Fig. 9. Detail of the two curves $\Gamma_{p, k}$ and $\Lambda_{p, k}$ associated with the end coil $\mathrm{C}_{p, k}$ for the computation of the internal self-inductance $L_{k}$. 
Section II where $\Gamma_{p, k}$ acts as the source turn (curve $\mathrm{A}^{\prime} \mathrm{B}^{\prime} \mathrm{C}^{\prime} \mathrm{D}^{\prime}$ in Fig. 2) and $\Lambda_{p, k}$ acts as the search turn (closed curve ABCD in Fig. 2). Hence:

$$
L_{k}^{(e x t)}=N_{k}^{2} M_{\Lambda_{p, k}-\Gamma_{p, k}}
$$

\section{B. Mutual inductance between distinct end coils}

The mutual inductance between two distinct end coils $\mathrm{C}_{p, k}$ and $\mathrm{C}_{p, k^{\prime}}$ is computed using the algorithms given in Section II where the source and search turns are the mean curves $\Gamma_{p, k}$ and $\Gamma_{p, k^{\prime}}$ respectively placed in the middle of the two mentioned end coils. Hence:

$$
M_{p, k, p^{\prime}, k^{\prime}}=N_{k} N_{k^{\prime}} M_{\Gamma_{p, k}-\Gamma_{p^{\prime}, k^{\prime}}}
$$

\section{APPLICATION EXAMPLE AND 3D FEA VALIDATION}

The analytical procedure described is herein applied to compute the end coil leakage inductance of a medium-voltage (MV) synchronous generator whose ratings are provided in Table II.

TABLE II. RATINGS OF THE MACHINE USED FOR VALIDATION

\begin{tabular}{c|c}
\hline \hline Rated power & $16.2 \mathrm{MVA}$ \\
\hline Rated voltage & $11 \mathrm{kV}$ \\
\hline Number of poles & 4 \\
\hline Rated speed & $1500 \mathrm{r} / \mathrm{min}$ \\
\hline Rated power factor & 0.85 \\
\hline Rated efficiency & 0.98 \\
\hline \hline
\end{tabular}

The field winding end coil structure is that shown in Fig. 1. The detailed dimensions and number of turns of all individual end coils (including the auxiliary curves $\Gamma_{p, k}$ and $\Lambda_{p, k}$ associated to them as explained in Section IV) are provided in Table III, which refers to the generic $p$-th pole of the machine (for the other poles, the data are the same). The nomenclature used in Table III to identify dimensions is the same introduced in Section II to characterize infinitely-thin end turns (Fig. 2). Regarding the end coil cross section dimensions (Fig. 8b), all end coils share the following sizes:

$$
w_{p, k}=21 \mathrm{~mm}, h_{p, k}=84 \mathrm{~mm}
$$

for any $p=1 . .4$ and $k=1 . .5$.

TABLE III. DIMENSIONS AND NUMBER OF TURNS FOR THE END COILS OF THE $p$ -

\begin{tabular}{c||c||c|c|c|c}
\multicolumn{1}{c||}{ Coil } & \multirow{2}{*}{ N. of turns } & Curve & $L[\mathrm{~mm}]$ & $R[\mathrm{~mm}]$ & $\phi_{2}-\phi_{1}[\mathrm{deg}]$ \\
\hline \hline \multirow{2}{*}{$\mathrm{C}_{p, 1}$} & \multirow{2}{*}{$N_{1}=6$} & $\Gamma_{p, 1}$ & 248 & 410 & 85 \\
\cline { 3 - 6 } & & $\Lambda_{p, 1}$ & 237 & 410 & 82 \\
\hline \multirow{2}{*}{$\mathrm{C}_{p, 2}$} & \multirow{2}{*}{$N_{2}=6$} & $\Gamma_{p, 2}$ & 204 & 410 & 75 \\
\cline { 3 - 6 } & & $\Lambda_{p, 2}$ & 193 & 410 & 72 \\
\hline \multirow{2}{*}{$\mathrm{C}_{p, 3}$} & \multirow{2}{*}{$N_{3}=6$} & $\Gamma_{p, 3}$ & 160 & 410 & 65 \\
\cline { 3 - 6 } & & $\Lambda_{p, 3}$ & 149 & 410 & 62 \\
\hline \multirow{2}{*}{$\mathrm{C}_{p, 4}$} & \multirow{2}{*}{$N_{4}=6$} & $\Gamma_{p, 4}$ & 116 & 410 & 55 \\
\cline { 3 - 6 } $\mathrm{C}_{p, 5}$ & \multirow{2}{*}{$N_{5}=6$} & $\Lambda_{p, 4}$ & 105 & 410 & 52 \\
\cline { 3 - 6 } & & $\Gamma_{p, 5}$ & 72 & 410 & 45 \\
\cline { 3 - 6 } & & $\Lambda_{p, 5}$ & 61 & 410 & 42
\end{tabular}

For the purpose of a numerical validation, the machine has been modeled for 3D magneto-static FEA. The meshed model used for this purpose is shown in Fig. 10 and does not have any damper winding. In order to assess the influence of

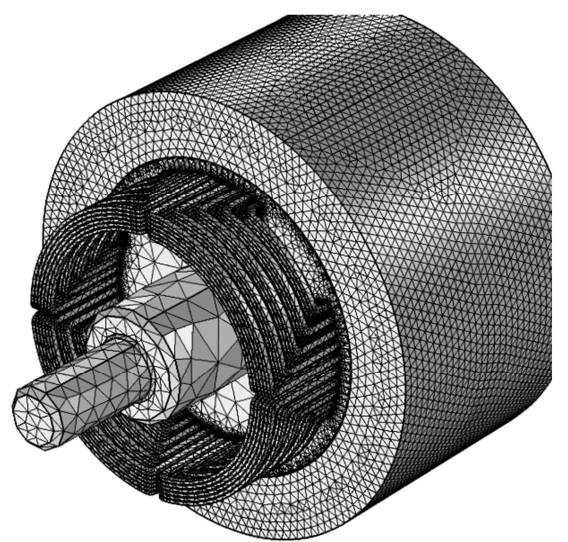

Fig. 10. Generator meshed model (without damper cage) used for magnetostatic 3D FEA.

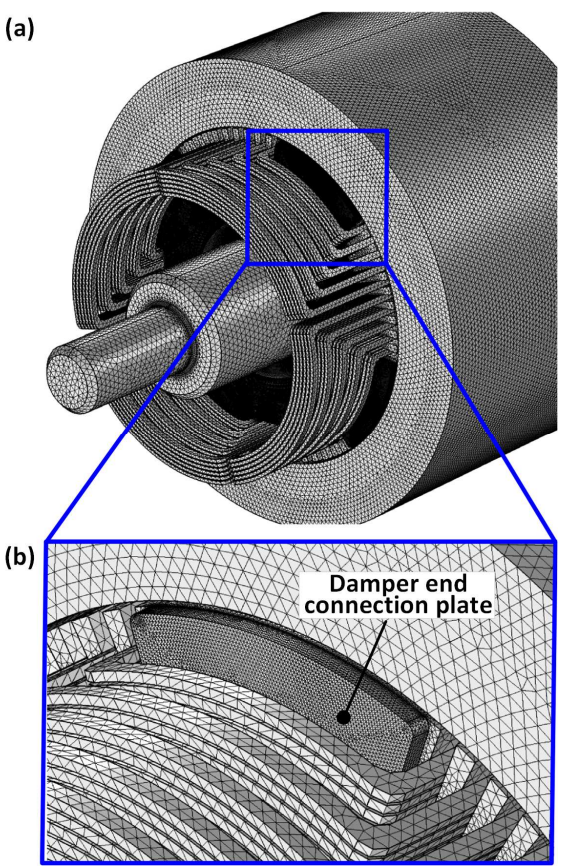

Fig. 11. Generator meshed model (with damper cage) used for timeharmonic 3D FEA.

ammortisseur circuits, which are generally mounted in the rotor, a time-harmonic 3D FEA simulation is also performed on a generator model equipped with damper windings as shown in Fig. 11. Fig. 11 shows that front aluminum plates are used as end connections to short circuit damper bars. The same components also act as clamping plates for the rotor laminated stack. End plates and damper bars need to be modeled with a small mesh size because eddy currents occur in them when the field is supplied by an AC current.

For the magnetostatic simulation, the field circuit is energized with its rated no-load DC current $(0 \mathrm{~Hz})$, while for the time-harmonic simulation the same current is applied with a frequency of $50 \mathrm{~Hz}$. The magnetostatic and time-harmonic FEA solutions in the core region are shown in Fig. 12a and $12 \mathrm{~b}$, respectively. It can be seen that, in presence of an AC field supply, a reaction current (e.g. $I_{d}$ in Fig. $12 \mathrm{~b}$ ) is induced in rotor dampers such that it weakens the air-gap flux 

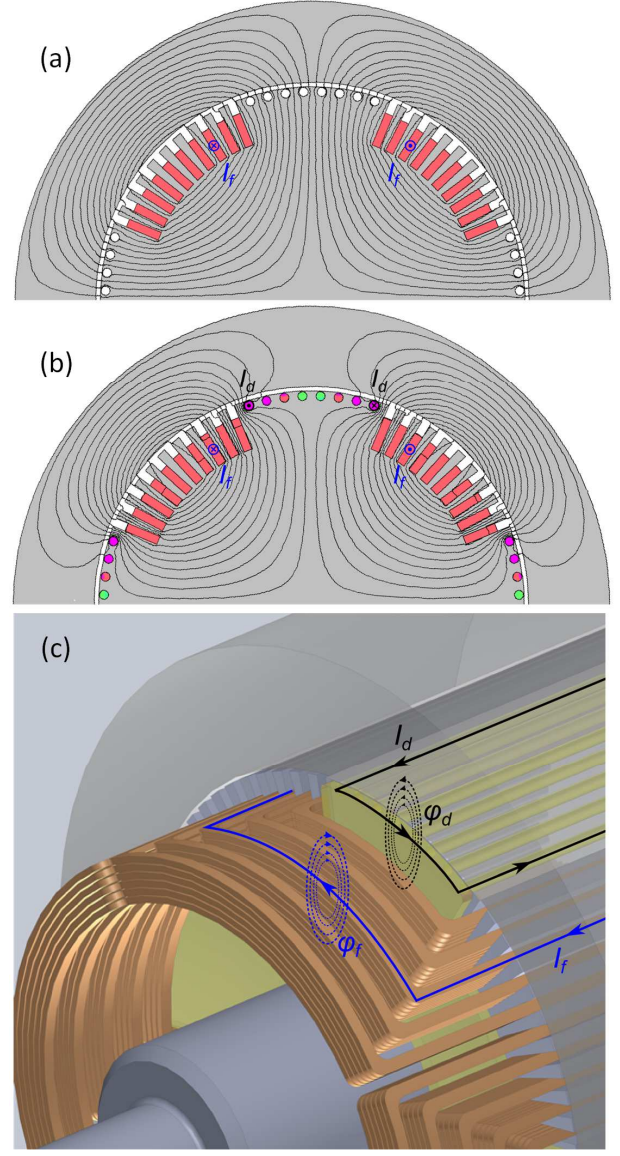

Fig. 12. (a) Magnetostatic solution in the core region. (b) Time-harmonic solution in the core region. (c) Schematic of field and damper currents with the relevant end leakage fluxes.

produced by the field current (e.g. $I_{f}$ in Fig. 12a and Fig. 12b) in accordance with Faraday-Lenz law. The leakage fluxes produced in the end region by the field current $\left(I_{f}\right)$ and by the damper current $\left(I_{d}\right)$ are qualitatively sketched in Fig. 12c. It can be noticed that the end leakage flux $\varphi_{d}$ due to the damper reaction current counteracts the end leakage flux $\varphi_{f}$ due to the field current as it happens in the core region (Fig. 12b). This is expected to result in a reduced field end-coil flux linkage when the damper is present and reactive.

For both the magnetostatic and the time-harmonic 3D FEA simulations, the field circuit end-coil leakage inductance is computed as:

$$
\lambda_{\text {field }} / I_{\text {field }}
$$

where $\lambda_{\text {field }}$ is the peak value of the total flux linked by all the end coils (at both rotor ends) and $I_{\text {field }}$ is the peak value of the field current. The results obtained are provided in Table IV.

TABLE IV. END COIL LEAKAGE INDUCTANCE COMPUTATION RESULTS

\begin{tabular}{l|c|c}
\hline \hline \multicolumn{2}{|c|}{ Analytical method } & $1.53 \mathrm{mH}$ \\
\hline \multirow{2}{*}{ 3D FEA } & Magnetostatic $(0 \mathrm{~Hz})$ & $1.57 \mathrm{mH}$ \\
\cline { 2 - 3 } & Time-harmonic $(50 \mathrm{~Hz})$ & $1.55 \mathrm{mH}$ \\
\hline \hline
\end{tabular}

The results shown in Table IV confirm that there is a slight reduction in the inductance obtained from the time-harmonic FEA as an effect of the end field due to damper reaction currents. However, the reduction is extremely small, proving that the end leakage flux produced by the reaction current in the damper end connection (schematically indicated as $\varphi_{d}$ in Fig. 12c) is very weakly linked by field-circuit end coils. It therefore appears that the damper cage does not have a significant influence on the field end-coil leakage inductance in the machine under study. However, this result is not claimed to be general as other kinds of damper cage construction (especially in regard to the end connection technology) may lead to different conclusions.

More importantly, what clearly appears from Table IV is that the leakage inductance value predicted analytically is in very good accordance with the results of 3D FEA simulations, with an estimation error in the order of $2 \%$. This is consistent with the good agreement already found in Section III in both experimental and 3D FEA assessments. However, while in Section III both the 3D FEA and the analytical model assumed an infinitesimal cross section for the end turns, in the validation example reported in this section the end coils have finite thickness and their actual rectangular cross section is approximated as circular so as to compute the internal leakage inductance according to (27). As already found by other authors [7], the approximation is demonstrated not to introduce a remarkable error since the internal inductance accounts for a small portion of the overall leakage inductance being evaluated. Moreover, in the 3D generator model shown in Fig. 10 and Fig. 11 there is an air gap (whose width is 5 $\mathrm{mm}$ ), which is not considered by the analytical model. In spite of this approximation, the analytical result exhibits a very good agreement with the 3D FEA simulation, confirming what has been already observed in Section III.B about the possibility to neglect air-gap effects without significant errors in the field circuit end-coil leakage inductance computation.

A further simplification underlying the results in Table IV is the fact that the analytical model disregards the ferromagnetic shaft which is included in the machine model for 3D FEA. This approximation is proved to introduce negligible errors, too, at least for the machine geometry under study (Fig. 10). The effect of a ferromagnetic shaft of different possible diameters is studied in more detail and in more general terms in the following Section.

\section{FERROMAGNETIC SHAFT EFFECTS ON THE END COIL INDUCTANCE}

In order to investigate the influence of a ferromagnetic shaft on the field winding end coil inductance, the 3D model shown in Fig. 13 is adopted. The situation is the same explored in Section II (Fig. 6) except that a ferromagnetic shaft of radius $R_{s}$ is included. The mutual inductance is computed by 3D FEA between the two end coils shown in Fig. 13, which are characterized by the data given in Table I. For clarity the average $R_{m}$ of the two end coil radii $R$ and $R^{\prime}$ is introduced:

$$
R_{m}=\left(R+R^{\prime}\right) / 2 .
$$

Fig. 14 shows the mutual inductance between the two end coils as a function of their displacement angle $\alpha$ and for different shaft radii $R_{s}$. It can be seen that some non-negligible effect occurs when the shaft radius exceeds $40 \%$ of the mean end coil radius $R_{m}$. 


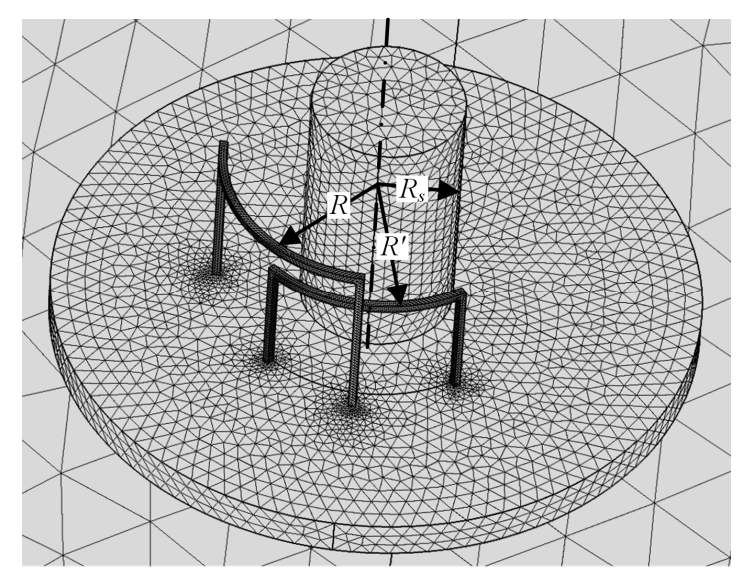

Fig. 13. 3D model used to study shaft effects on the mutual inductance between end coils.

The shaft effect on end coil leakage inductance values is further clarifier in Fig. 15, where the mutual inductance between two end coils, fixed in a given mutual position with displacement angle $\alpha$, is plotted versus the shaft radius.

In the case study addressed in Section V (Fig. 10), the shaft radius is significantly smaller than $40 \%$ of the mean end coil radius, hence shaft effects can be reasonably neglected in the analytical calculations without introducing remarkable errors as proven by the good accordance found between computation and FEA results (Table IV). For different geometries where the shaft is ferromagnetic and features a larger size compared to the mean field winding diameter, it may be possible that end coil leakage inductances can be influenced by the presence of the shaft to a larger extent and a 3D FEA simulation may be required for a more accurate estimation. Further studies on how to incorporate shaft effects in the analytical computation based on the method of mirror images are presently in progress.
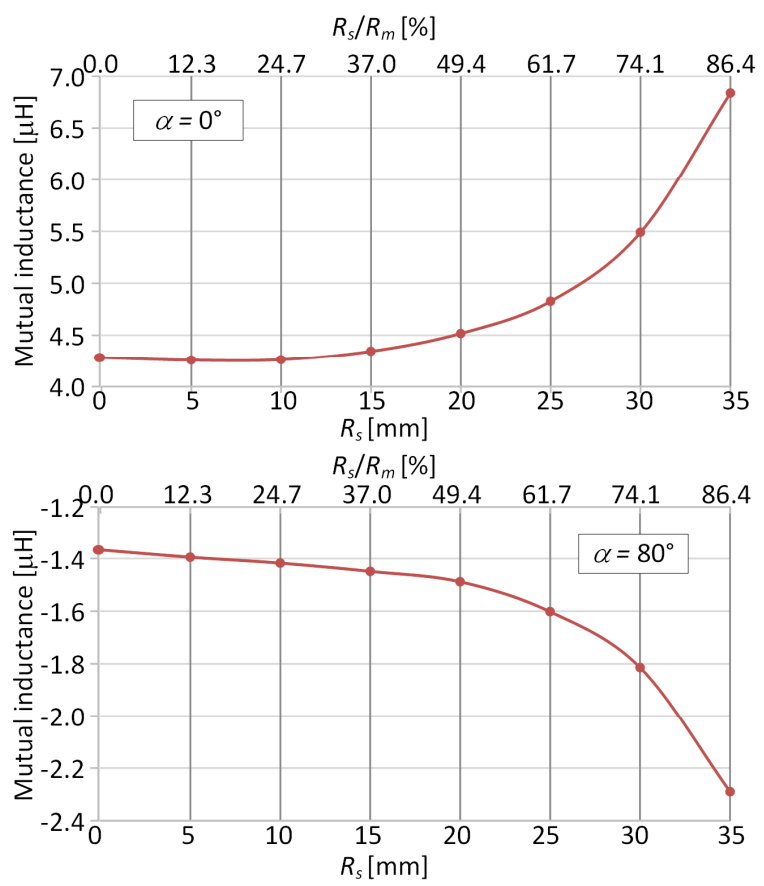

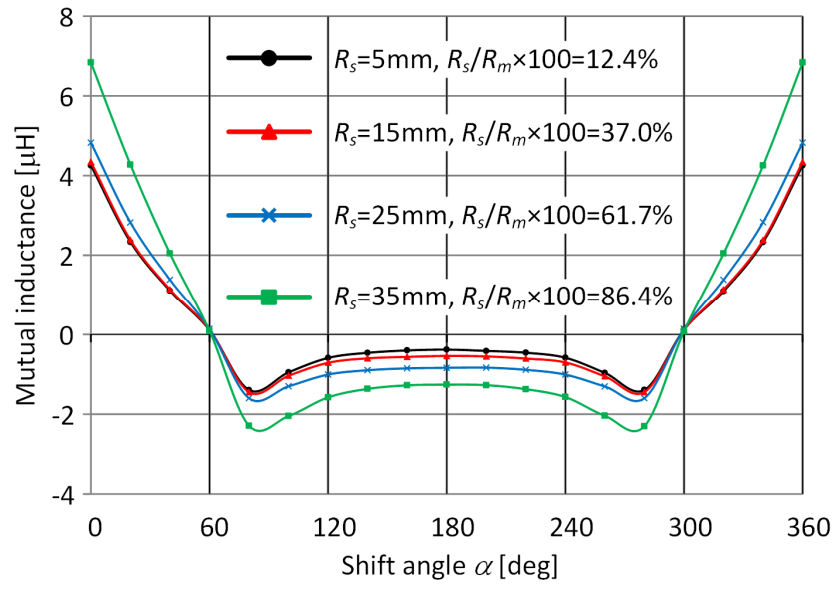

Fig. 14. Mutual inductance between two end coils as a function of their relative displacement, for different ferromagnetic shaft radii.

\section{COMPARISON BETWEN THE PROPOSED AND EXISTING} METHODS FOR END-COIL LEAKAGE INDUCTANCE COMPUTATION

The presented method for the computation of field-circuit end-coil leakage inductances is basically a fully-analytical version of the hybrid (numerical-analytical) technique proposed in [7]-[10], where end coils are discretized into elementary straight segments so as to compute their self and mutual inductances through Neumann integrals and the method of mirror images. Therefore, the level of accuracy achievable with the proposed method is essentially the same as for the method discussed in [7]-[10], the main advantage lying in the time and computation resource savings that result from a merely analytical approach where no domain discretization is required.

Approximated algebraic formulas can be found in the

$$
R_{s} / R_{m}[\%]
$$
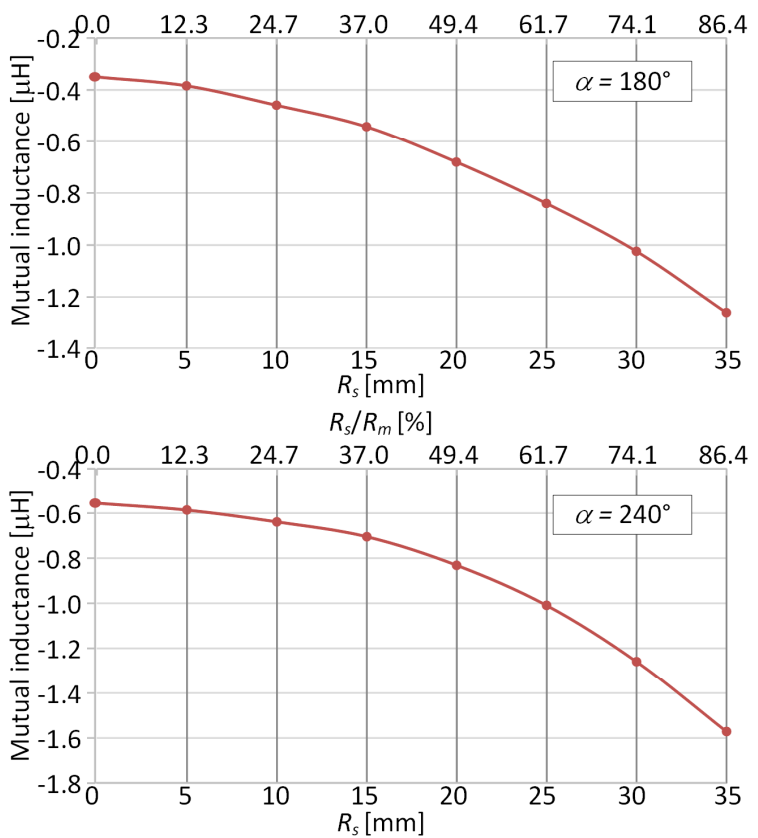

Fig. 15. Mutual inductance between two end coils as a function of the ferromagnetic shaft radius, for different values of the relative displacement angle between end coils. 
technical literature for a first-attempt guess of field circuit end-coil leakage inductances, as well. Examples can be found in [14], [15] where empirical expressions are provided based on experimentally-determined coefficients found through tests on reduced-scale simplified prototypes. As clearly stated in [14], however, these approximate formulas have poor reliability and are expected to significantly underestimate end coil leakage inductance actual values for full-scale synchronous machines. Just to give a numerical example, [16] proposes the following expression to estimate the field-circuit end-coil leakage inductance of cylindrical-rotor synchronous machines:

$$
L_{t o t}=P \mu_{0} N_{f p} W_{f c} l_{p} \lambda_{e f},
$$

where $P$ is the number of poles, $N_{f p}$ is the number of fieldwinding slots per pole, $W_{f c}$ is the number of turns per rotor slots, $l_{p}$ is the total rotor length and $\lambda_{e f}$ is a permeance coefficient. The evaluation of $\lambda_{e f}$ is proposed as follows:

$$
\lambda_{\text {ef }}=0.2 \tau_{r} / l_{i},
$$

where $\tau_{r}$ is the average coil pitch for the field winding coils and $l_{i}$ is the ideal core length [16]. In the case of the machine considered in Section $\mathrm{V}$ for 3D FEA validation, we have: $P=4$, $N_{f p}=10, W_{f c}=6, l_{p}=1.6 \mathrm{~m}, l_{i}=1.48 \mathrm{~m}, \tau_{r}=0.93 \mathrm{~m}$. With such parameters, $L_{t o t}$ is estimated by (33) to be $0.36 \mathrm{mH}$, which appears significantly smaller that the values obtained analytically and by 3D FEA (Table IV).

\section{CONCLUSION}

In this paper, a fully analytical approach has been proposed for computing the end coil leakage inductance of the field winding in round-rotor synchronous machines. The proposed method descends from the analytical solution of Neumann integrals combined with the method of mirror images to account for stator and rotor magnetic core effects. As a first step, analytical formulas have been provided to compute the mutual inductance between single end turns and such formulas have been validated against both 3D FEA simulations and experimental measurements on a dedicated laboratory setup. Then, the validated equations have been used to compute the overall field winding end coil leakage inductance and computation results have been assessed by comparison with 3D FEA. Finally, the influence of a ferromagnetic shaft on the inductance computation has been investigated using 3D FEA. It has been demonstrated that, when the shaft radius is smaller than $40 \%$ of the mean winding radius, shaft effects can be disregarded with negligible errors. Further studies are presently in progress on how to account for the mentioned shaft effects in the analytical computation of field winding end coil leakage inductance.

\section{REFERENCES}

[1] K. C. Kim, H. W. Lee, Y. D. Chun, J. Lee, "Analysis of electromagnetic force distribution on end winding for motor reliance", IEEE Trans. on Magnetics, vol. 41, no. 10, Oct. 2005, pp. 4072-4074.

[2] Taieb Brahimi, A. Foggia, G. Meunier, "End winding reactance computation using a 3D finite element program", IEEE Trans. on Magnetics, vol. 29, no. 2, March 1993, pp. 1411-1414.

[3] T. Cox, F. Eastham, J. Proverbs, "End Turn Leakage Reactance of Concentrated Modular Winding Stators", IEEE Transactions on Magnetics, vol.44, no.11, pp.4057-4061, Nov. 2008.
[4] F. W. Grover, Inductance Calculations, pp. 45-47, Dover Phoenix Editions, 2004.

[5] C. J. Carpenter, "The application of the method of images to machine end-winding fields", Proceedings of IEE, vol. 107, pt. A, 1960, pp. 487-500.

[6] Y. Liang, X. Bian, H. Yu, L. Wu, B. Wang, "Analytical Algorithm for Strand End Leakage Reactance of Transposition Bar in AC Machine," IEEE Transactions on Energy Conversion, early access article.

[7] D. Ban, D. Zarko, I. Mandic, "Turbogenerator end-winding leakage inductance calculation using a 3-D analytical approach based on the solution of Neumann Integrals," IEEE Transactions on Energy Conversion, vol.20, no.1, pp.98-105, March 2005.

[8] A. Tessarolo, F. Luise, "An Analytical-Numeric Method for Stator End-Coil Leakage Inductance Computation in Multi-Phase Electric Machines", IEEE Industry Applications Society Annual Meeting, pp.18, 5-9, Oct. 2008.

[9] M. Freese, "Analytic calculation of turbo generator end winding inductances using Neumann's formula”, International Symposium on Power Electronics Electrical Drives Automation and Motion, SPEEDAM 2010, pp. 1597-1602, 14-16 June 2010.

[10] A. Tessarolo, "Computing end-turn leakage inductance of concentric coil windings by asymptotical series approximation of Neumann integrals", 2010 XIX International Conference on Electrical Machines (ICEM), pp.1-6, 6-8 Sept. 2010.

[11] G. A. Campbell, "Mutual inductances of circuits composed of straight wires", Physical Review, vol. 5, 1915, pp. 452-458.

[12] A. Tessarolo, C. Bassi, "Stator Harmonic Currents in VSI-Fed Synchronous Motors With Multiple Three-Phase Armature Windings," IEEE Transactions on Energy Conversion, vol.25, no.4, pp.974-982, Dec. 2010.

[13] G. B. Arfken, H. J. Weber, Mathematical Methods for Physicists, Academic Press, 2001, pp. 740-742, pp. 772-777, pp. 786-790.

[14] W. Schuisky, Berechnung elektrischer Maschinen, Verlag Springer, 1960.

[15] Weissheimer, Die Stirnstreuung der Synchronmaschinen. Arbeiten aus dem Elektrotechnischen Institut der Tecnischen Hochschule Karlsruhe, Band 5 (1927-1929).

[16] Ion Boldea, The Electric Generator Handbook, CRC Press, 2006. 